\title{
Ciclização intramolecular: uma estratégia promissora no desenvolvimento de pró-fármacos
}

\author{
Cledir Santos* \\ Departamento de Química Fundamental, Universidade Federal de Pernambuco
}

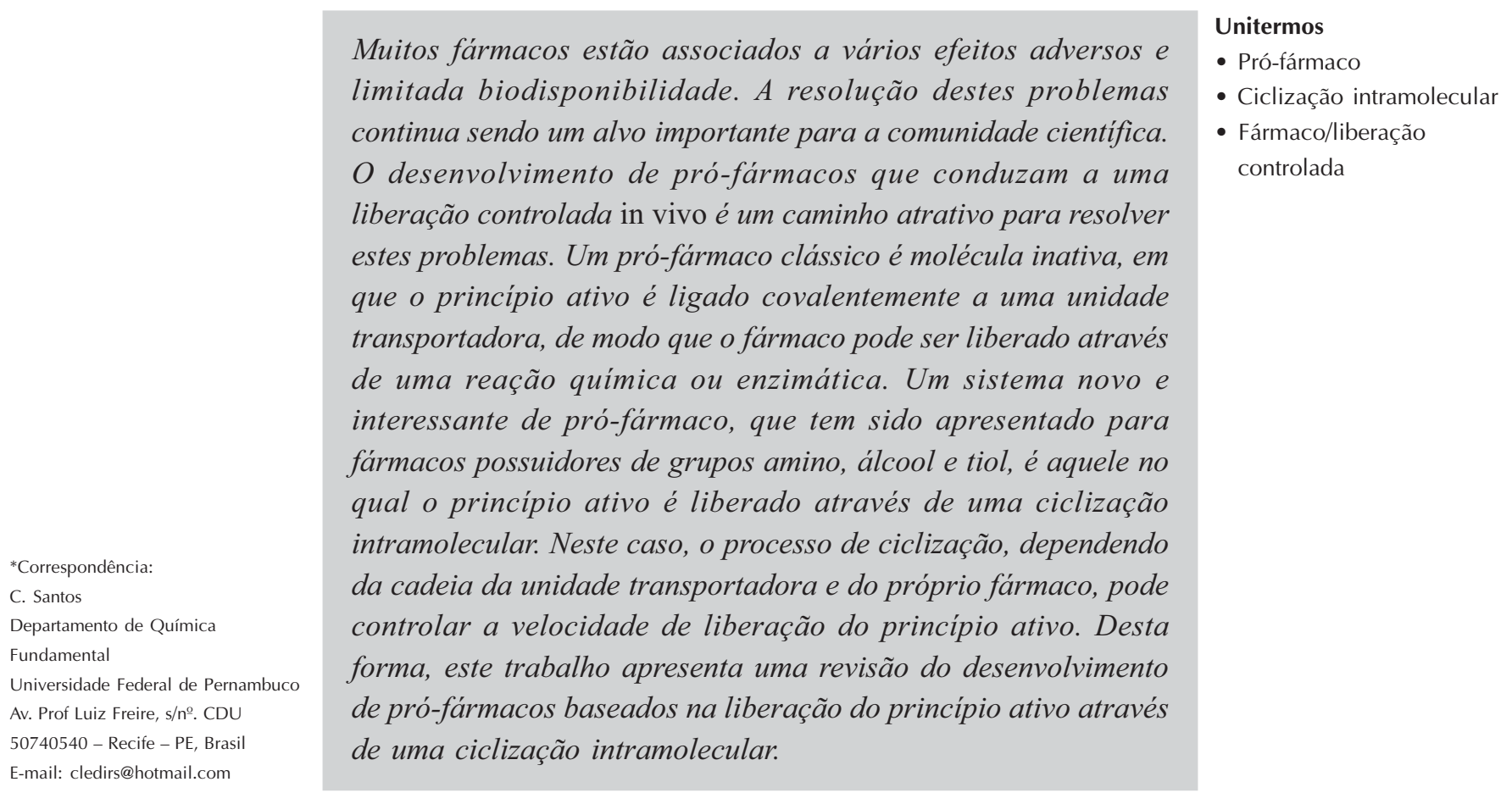

\section{INTRODUÇÃO}

Muitos fármacos que apresentam elevada atividade contra várias doenças estão relacionados com problemas que os tornam limitados ou inviáveis como sistemas terapêuticos eficazes (Guettari et al., 1997; Song et al., 2005). Solubilidade, estabilidade, absorção, distribuição, toxicidade e biodisponibilidade são aspectos fundamentais a serem levados em consideração quando da administração de um fármaco (Lynx et al., 2006; Otto, 2004; Vennerstrom et al., 1999; Thomsen et al., 2004). Um exemplo comum de fármacos que estão relacionados com vários problemas de natureza terapêutica é encontrado nos análogos das purinas e pirimidinas. Os fármacos contidos nestas classes de compostos são conhecidos pelas suas baixas biodisponibilidades orais (Thomsen et al., 2003). O aciclovir, por exemplo, é um análogo purínico que apresenta uma biodisponibilidade oral de cerca de 20\% em adultos (Steingrimsdottir et al., 2000). Já a fluorouracila, que é um análogo pirimidínico, apresenta uma biodisponibilidade oral que varia entre indivíduos numa escala de 0-80\% (Guo et al., 1995; Heyden et al., 1999; Zinutti et al., 1998). Além da reduzida biodisponibilidade destes fármacos, problemas associados à elevada toxicidade e baixa solubilidade, tanto em meio aquoso quanto em meio lipofílico, são um verdadeiro entrave nas suas aplicações terapêuticas (De Vrueh et al., 1998; Han et al., 1998; Zambonin et al., 1998).

Um medicamento acabado é fruto de décadas de pesquisa química, farmacêutica e clínica. No entanto, muitas vezes subsistem problemas como os anteriormente menci- 
onados. Uma estratégia largamente utilizada para solucionar problemas causados pela utilização de determinados fármacos tem sido o desenvolvimento de pró-fármacos. $\mathrm{O}$ termo "pró-fármaco" foi estabelecido por Adrien Albert em 1958 (Albert, 1958), justamente para designar compostos que sofrem biotransformação antes de exercerem a sua atividade farmacológica, comportando-se como agentes terapêuticos inativos per se, mas que são transformados no organismo para gerar um ou mais metabolitos ativos. Contudo, somente após 1970, quando foi possível investigar o destino dos fármacos no organismo de modo a compreender melhor a absorção, o metabolismo, a distribuição e a excreção, é que a utilização de pró-fármacos, como sistemas de liberação do princípio ativo, tomou grande impulso (Chung, Ferreira, 1999). Nas últimas décadas, um elevado número de trabalhos tem contribuído para o desenvolvimento de uma larga variedade de pró-fármacos, conduzindo, em muitos casos, a melhorias significativas nas propriedades farmacocinéticas e farmacodinâmicas dos fármacos parentais (Bundgaard, 1991; Siccardi et al., 2004; Sriram et al., 2004; Zheng et al., 1999).

Atualmente, os pró-fármacos podem ser classificados segundo dois critérios (Ettmayer et al., 2004):

1. Pela classe química:

a) pró-fármacos clássicos;

b) bioprecursores;

c) sistema químico planejado para liberação do fármaco no local de ação;

d) pró-fármacos macromoleculares;

e) conjugados fármaco-anticorpo.

2. Pelo mecanismo de ativação:

a) enzimático;

b) não-enzimático ou químico.

Apesar de, até há relativamente pouco tempo, a utilização de pró-fármacos ativados pela via química se apresentar como um campo pouco explorado, nos últimos anos tem atraído um elevado número de adeptos (Saari et al., 1990; Shan et al., 1997; Testa, Mayer, 1998). A crescente busca deste tipo de pró-fármacos é explicada pelo fato de, nos casos de ativação pela via enzimática, a liberação do fármaco estar sujeita à variabilidade da atividade enzimática entre espécies ou entre indivíduos de mesma espécie. Essa variabilidade produz grandes diferenças na velocidade de liberação do princípio ativo, de indivíduo para indivíduo (Saari et al., 1990; Shan et al., 1997), comprometendo a previsibilidade destes parâmetros e tornando o pró-fármaco um sistema pouco atrativo para liberação do princípio ativo in vivo.
Hoje, conta-se com um largo número de modelos de derivados de fármacos ativados quimicamente, nos quais a liberação do princípio ativo é uma função da estabilidade química da ligação fármaco-transportador (Andersen et al., 2006; Steffansen et al., 2005; Friedrichsen et al., 2001; Testa, Mayer, 1998) em determinado local de ação terapêutica.

Dentre os vários mecanismos de ativação química de pró-fármacos já disponíveis, o que se apresenta como um exemplo promissor é aquele em que o fármaco é liberado através de uma ciclização intramolecular que, contudo, é ainda pouco explorada (Shan et al., 1997; Testa, Mayer, 1998). De um modo geral, um pró-fármaco ativado por ciclização intramolecular possui um grupo nucleófilo capaz de atacar intramolecularmente um grupo eletrófilo, conduzindo à ciclização do sistema transportador e à quebra da ligação entre este e o princípio ativo como uma dependência do $\mathrm{pH}$ e da temperatura do meio, (Fredholt et al., 1995; Matsumoto et al., 2001 a; Thomsen, Bundgaard, 1993), como exemplificado no Esquema 1.

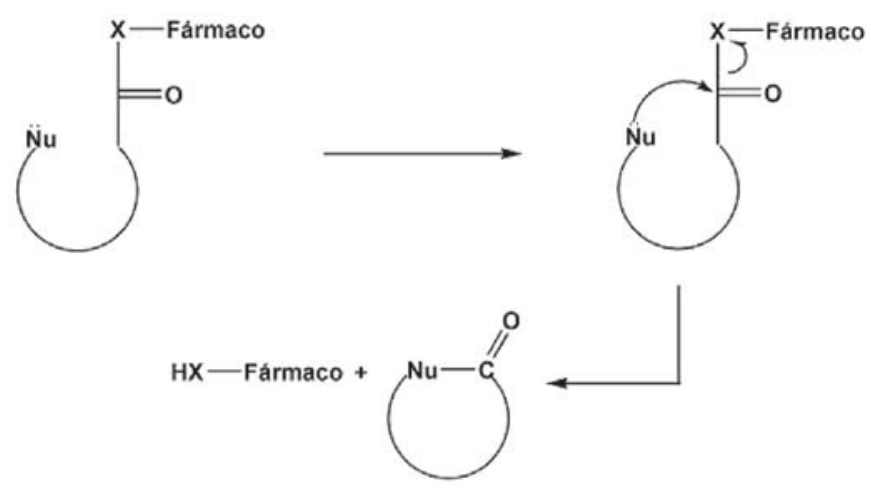

ESQUEMA 1 - Ativação de um pró-fármaco por ciclização intramolecular promovida por ataque de um grupo nucleófilo $(\mathrm{Nu})$ do transportador a um grupo eletrófilo (carbonila) presente na ligação fármacotransportador ( $\mathrm{X}=-\mathrm{NH}-$, -O- ou -S-).

\section{Os íons fenolato e alcoolato como agentes nucleofílicos}

Os grupos fenolato e alcoolato têm surgido como importantes nucleófilos no desenvolvimento de prófármacos ativados por ciclização intramolecular (Bundgaard et al., 1986; Larsen et al., 2004; Vigroux et al., 1995). No entanto, são escassos os dados da literatura acerca da ativação de pró-fármacos pelo uso desta estratégia em particular. Dentre alguns exemplos já conhecidos de derivados de fármacos ativáveis por esse mecanismo, podem-se destacar os obtidos de acordo com o modelo pro- 
posto por Vigroux et al. (1995). Este modelo fundamentase no desenvolvimento de potenciais pró-fármacos baseados em derivados fenólicos e alcoólicos de carbamatos.

Uma larga variedade desses compostos foi analisada quanto à sua estabilidade química e enzimática, conduzindo a resultados promissores no sentido da obtenção de prófármacos ativáveis por ciclização intramolecular e enzimaticamente estáveis, com a possibilidade de modular as propriedades estereoeletrônicas dos substituintes do anel aromático (Vigroux et al., 1995). Assim, a liberação do fármaco, a partir deste tipo de derivado, pode ocorrer através de uma ciclização intramolecular pelo ataque nucleofílico do íon fenolato sobre o grupo carbonílico, como apresentado no Esquema 2.
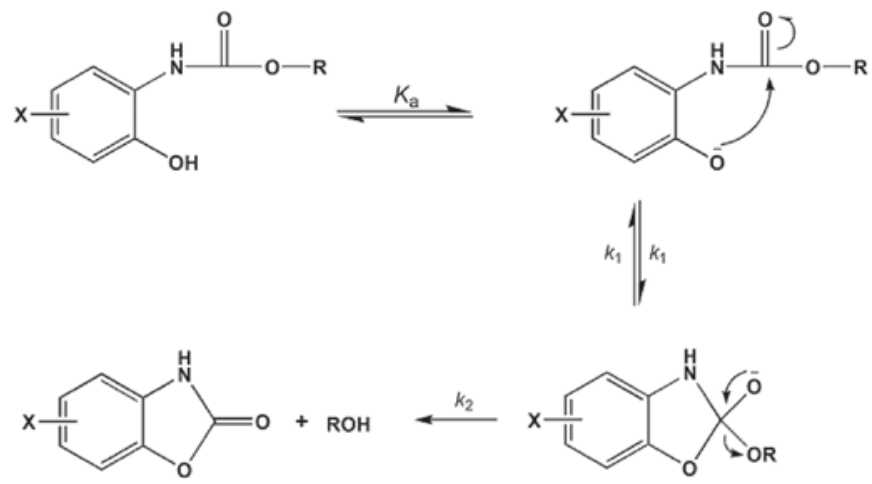

ESQUEMA 2 - Liberação de um fármaco alcóolico ou fenólico $(\mathrm{ROH})$ por ciclização intramolecular a partir de derivados propostos por Vigroux et al. (1995) $(\mathrm{X}=\mathrm{Cl}$ ou grupo benzila).

A utilização deste tipo de sistema transportador pode representar uma vantagem adicional frente a outros tipos conhecidos, pois dependendo das posições adotadas pelos grupos substituintes do anel aromático, o produto de ciclização, por si, pode possuir atividade farmacológica. Assim, a sua utilização como sistema de liberação do fármaco pode constituir um caso de "pró-fármaco mútuo". O termo "pró-fármaco mútuo" descreve a associação, numa única molécula, de dois fármacos normalmente com efeito sinérgico, ligados um ao outro através de uma ligação covalente (Carvalho et al., 2000). Um exemplo de prófármaco mútuo do paracetamol (1) foi observado no caso em que foi adicionado um átomo de cloro na posição 5 do anel aromático do transportador, conforme descrito no Esquema 2. Para este caso, a liberação quantitativa do paracetamol em $\mathrm{pH} 7,4$ e $37^{\circ} \mathrm{C}$ foi atribuída a uma ciclização intramolecular, conseqüente à formação também quantitativa do 5-clorobenzo[d]-oxazolidin-2-ona, que é um relaxante músculo-esquelético (2).

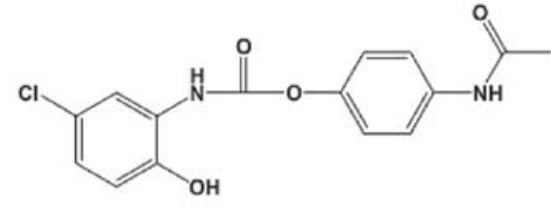

1



2
De um modo geral, os resultados deste trabalho indicaram que a reatividade dos compostos concebidos a partir deste modelo foi fortemente influenciada pelo valor do $\mathrm{p} K_{\mathrm{a}}$ do grupo abandonador. Para o composto 1, por exemplo, que tem como grupo abandonador o paracetamol $\left(\mathrm{p} K_{\mathrm{a}}=9,51\right)$ (Dechtlaruk et al., 1976) obteve-se um tempo de meia-vida de cerca de 7,1 segundos, a $37^{\circ} \mathrm{C}$ e $\mathrm{pH} 7,4$. Por outro lado, para o composto 3 , que tem o etanol $\left(\mathrm{p} K_{\mathrm{a}}=16,0\right)$ (Michigan State University, 2007) como grupo abandonador, obteve-se um tempo de meia-vida da ordem de 290 dias, nas mesmas condições de $\mathrm{pH}$ e temperatura.

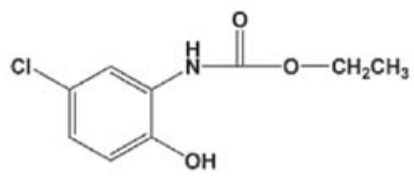

3

Um modelo de pró-fármacos ativados intramolecularmente graças ao caráter nucleofílico do grupo alcoolato é encontrado no sistema transportador apresentado por Bundgaard et al. (1986) (4, Esquema 3). Este sistema foi desenvolvido com o objetivo de melhorar a baixa biodisponibilidade ocular (1-3\%) da pilocarpina (5), um fármaco largamente utilizado para controlar a elevada pressão intraocular associada ao glaucoma. Através deste modelo, o fármaco (5) é gerado por uma ciclização intramolecular com concomitante liberação da unidade transportadora, como apresentado pelo Esquema 3.

Uma larga variedade de ésteres alquílicos e aralquílicos (4) derivados do ácido pilocárpico foram sintetizados e avaliados como sistemas passíveis de sofrer ciclização intramolecular conducente à liberação do princípio ativo (5). A reatividade dos compostos obtidos foi analisada em diversos tampões aquosos, a $37^{\circ} \mathrm{C}$, numa extensa escala de $\mathrm{pH}$ (Bundgaard et al., 1986). A análise detalhada dos produtos de hidrólise nessas condições indicou que todos os substratos sofreram ciclização intramolecular com formação quantitativa do princípio ativo. Em relação ao mecanismo de ciclização intramolecular dos diferentes ésteres do ácido pilocárpico, foi observado que a reação ocorreu através de um pré-equilíbrio de ionização do grupo hidroxila (Esquema 3), seguido pelo ataque nucleofílico do íon alcoolato - formado no 


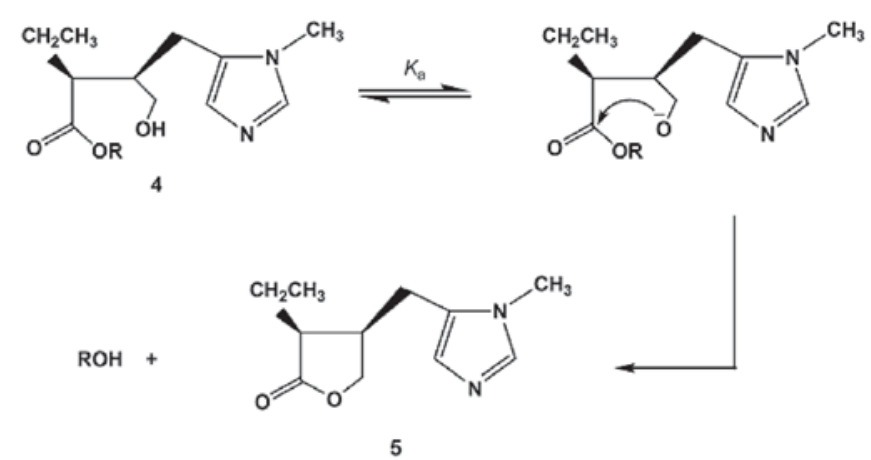

ESQUEMA 3 - Modelo de pró-fármaco (4) proposto por Bundgaard et al. (1986) para a liberação de pilocarpina (5) e do sistema transportador ROH (derivados alquílicos e aralquílicos do ácido pilocárpico).

referido equilíbrio - sobre o grupo éster. A análise dos dados obtidos neste trabalho sugere que o aparecimento deste pré-equilíbrio foi devido à ocorrência de aparente catálise pelo íon hidróxido, pois a velocidade de ciclização intramolecular aumentou proporcionalmente com a atividade deste íon na escala de $\mathrm{pH}$ estudada.

A ciclização intramolecular dos compostos analisados foi também uma função do efeito estérico e polar das cadeias alcoólicas dos ésteres, sublinhando a versatilidade do modelo apresentado. Assim, estes compostos surgem como promissores pró-fármacos para o transporte e liberação controlada do princípio ativo. Esta afirmação pode ser justificada pela análise da variação dos tempos de meiavida obtidos para o mais instável e para o mais estável dos compostos, que foram os que possuíam como substituintes os grupos $p$-clorobenzila $\left(6, \mathrm{t}_{1 / 2}=30\right.$ minutos $)$ e $n$-hexila $\left(7, \mathrm{t}_{1 / 2}=1105\right.$ minutos), quando analisados em tampão aquoso a $37^{\circ} \mathrm{C} \mathrm{e} \mathrm{pH} \mathrm{7,4.} \mathrm{Outro} \mathrm{fator} \mathrm{relevante} \mathrm{na} \mathrm{análise}$ desses resultados reside no fato de que todos os ésteres sintetizados se apresentaram visivelmente mais lipofílicos que o próprio fármaco. De modo geral, estes dados sugerem que os ésteres do ácido pilocárpico são fortes candidatos a prófármacos da pilocarpina e que este modelo de pró-fármaco pode ser otimizado e expandido para o transporte e liberação de outros fármacos por ciclização intramolecular ao princípio ativo ou a um derivado cíclico do sistema transportador, neste caso com concomitante liberação do princípio ativo.

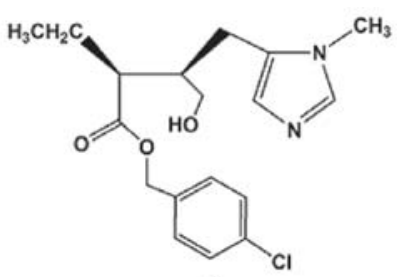

6

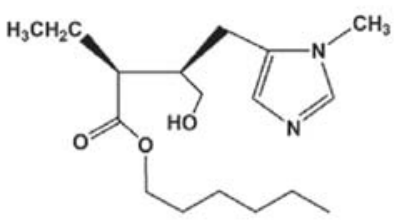

7

\section{O íon carboxilato como agente nucleofílico}

O grupo carboxilato também tem sido estudado como um agente nucleofílico no desenvolvimento de prófármacos ativados por ciclização intramolecular (Fredholt et al., 1995; Kovach et al., 1981). Assim como para o caso dos potenciais pró-fármacos ativados pelo ataque do íon alcoolato ou fenolato sobre a ligação éster, os dados da literatura referentes aos modelos de potenciais pró-fármacos ativados pelo ataque nucleofílico do íon carboxilato sobre a ligação éster também são escassos. Fredholt et al. (1995) desenvolveram um modelo de potenciais pró-fármacos de fármacos fenólicos, ativados por ciclização intramolecular, com a finalidade de proteger o princípio ativo do efeito de metabolismo pré-sistêmico, que promove a eliminação do fármaco antes de promover a sua atividade biológica, o que ocorre para um largo número de fármacos fenólicos administrados pela via oral. Assim, vários derivados resultantes da ligação de diferentes ácidos dicarboxílicos ao paracetamol e ao fenol foram sintetizados e avaliados quanto à sua estabilidade in vitro e quanto ao mecanismo de liberação do resíduo fenólico. Na Tabela I apresentam-se os vários derivados de ambos os fenóis, obtidos pelos autores acima referidos.

TABELA I - Derivados do paracetamol (8-12) e do fenol (13-16) propostos por Fredholt et al. (1995)
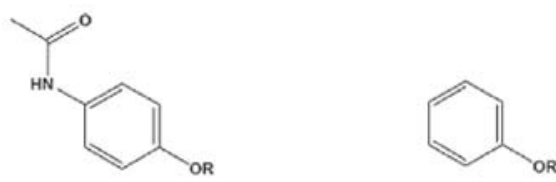

\begin{tabular}{llrl}
\hline Composto & \multicolumn{1}{c}{$\mathrm{R}$} & \multicolumn{1}{c}{ Composto } \\
\hline $\mathbf{8}$ & $-\mathrm{COCH}_{2} \mathrm{CH}_{2} \mathrm{CH}_{2} \mathrm{COOH}$ & $\mathbf{1 3}$ & $-\mathrm{COCH}_{2} \mathrm{CH}_{2} \mathrm{CH}_{2} \mathrm{COOH}$ \\
$\mathbf{9}$ & $-\mathrm{COCH}_{2} \mathrm{CH}_{(}\left(\mathrm{CH}_{3}\right) \mathrm{CH}_{2} \mathrm{COOH}$ & $\mathbf{1 4}$ & $\left.-\mathrm{COCH}_{2} \mathrm{CH}_{(} \mathrm{CH}_{3}\right) \mathrm{CH}_{2} \mathrm{COOH}$ \\
$\mathbf{1 0}$ & $\left.-\mathrm{COCH}_{2} \mathrm{C}_{(} \mathrm{CH}_{3}\right)_{2} \mathrm{CH}_{2} \mathrm{COOH}$ & $\mathbf{1 5}$ & $-\mathrm{COCH}_{2} \mathrm{OCH}_{2} \mathrm{COOH}$ \\
$\mathbf{1 1}$ & $-\mathrm{COCH}_{2} \mathrm{OCH}_{2} \mathrm{COOH}$ & $\mathbf{1 6}$ & $-\mathrm{COCH}_{2} \mathrm{CH}_{2} \mathrm{COOH}$ \\
$\mathbf{1 2}$ & $-\mathrm{COCH}_{2} \mathrm{CH}_{2} \mathrm{COOH}$ & & \\
\hline
\end{tabular}

A reatividade destes compostos foi analisada em vários tampões, a $37^{\circ} \mathrm{C}$, numa larga escala de $\mathrm{pH}$, onde se observou que a liberação, tanto do paracetamol quanto do fenol, foi quantitativa para todos os valores de $\mathrm{pH}$ estudados. Os perfis de $\mathrm{pH}$-velocidade para a degradação dos derivados $\mathbf{1 0}$ e 14, apresentados na Figura 1, mostram que a máxima estabilidade destes compostos foi observada na região de $\mathrm{pH}$ entre 2 e 5 . Esta evidência experimental é devida ao fato da extensão de ionização dos grupos carboxílicos ser baixa nessa região de $\mathrm{pH}$ tornando-os, assim, fracos agentes nucleofílicos (Peterson lab, 2007). 


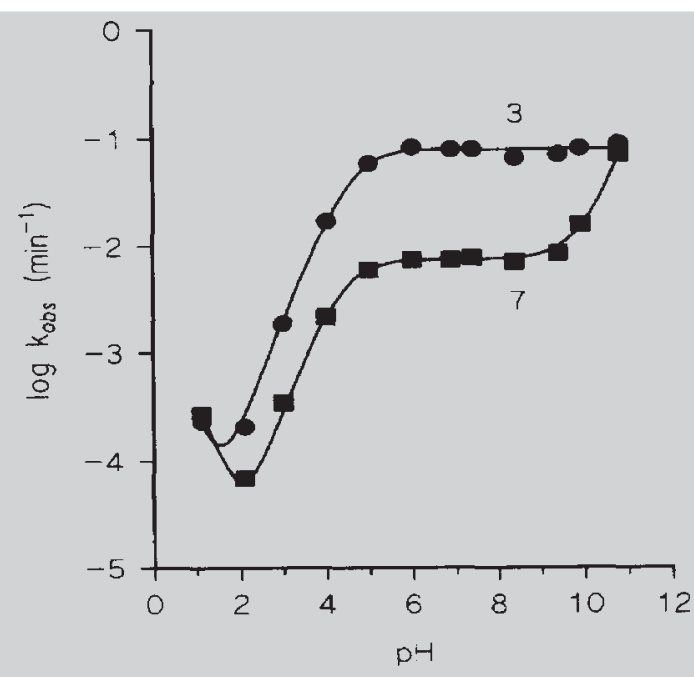

FIGURA 1 - Perfis de pH-velocidade para a degradação dos compostos $\mathbf{1 0}(\bullet)$ e $\mathbf{1 4}(\bullet)$ em meio aquoso a $37^{\circ} \mathrm{C}$.

Segundo os autores, a liberação do paracetamol e do fenol, a partir dos derivados em questão, ocorreu por ciclização intramolecular devido ao ataque do grupo carboxilato sobre a ligação éster. No entanto, os autores concluíram que, paralelamente à ciclização intramolecular, ocorreu hidrólise ácida ou básica da ligação éster, conduzindo à formação do respectivo ácido dicarboxílico e do fenol, como apresentado no Esquema 4 para a degradação do composto 14. Neste esquema, observa-se que o ataque nucleofílico intramolecular resultou na formação do fenol e do respectivo anidrido, que é posteriormente degradado ao correspondente ácido dicarboxílico.

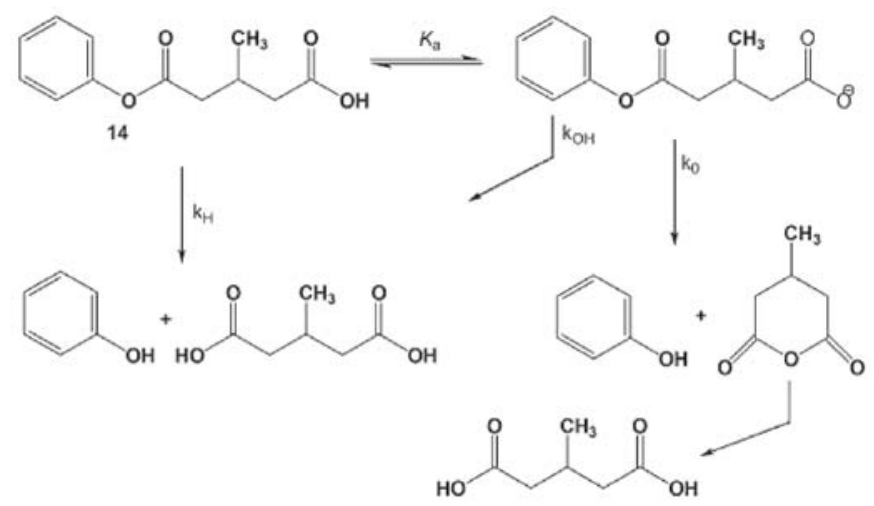

ESQUEMA 4 - Diferentes vias de liberação do fenol a partir do seu derivado 14.

A análise da reatividade de todos os compostos a $\mathrm{pH}$ 7,4 e $37^{\circ} \mathrm{C}$ mostrou que, nessas condições, a ciclização intramolecular foi a reação que mais contribuiu para a liberação das unidades fenólicas. A observação dos tempos de meia-vida obtidos para a degradação de cada derivado indicou que a reatividade dos compostos 8-16 foi uma função de três fatores: 1) tamanho da cadeia principal dos ácidos dicarboxílicos; 2) número de substituintes presentes nessa cadeia e 3 ) valor do $\mathrm{p} K_{\mathrm{a}}$ do grupo fenólico.

Para os derivados do paracetamol que possuíam como unidade transportadora o ácido butanodióico (12), a velocidade de reação foi cerca de 133 vezes superior àquela observada quando a unidade transportadora era o ácido pentanodióico (8). O mesmo fato foi observado para ambos os derivados homólogos do fenol, com uma diferença de reatividade em torno de 151 vezes. A elevada reatividade dos compostos que têm o ácido butanodióico como unidade transportadora, frente aos que têm o ácido pentanodióico, é atribuída à formação de anéis de quatro membros que, neste caso, são mais reativos (Bruice et al., 1960).

A diferença estrutural entre o derivado 8 e o derivado 9 está na presença de um grupo metila lateral existente neste último. Esta alteração estrutural gera uma diferença de reatividade de 3,8 vezes, ou seja, o derivado ramificado (9) é cerca de 3,8 vezes mais reativo que o não ramificado (8). Por outro lado, quando existem dois grupos metila laterais (10), a reatividade é aumentada em cerca de 18 vezes relativamente ao composto não ramificado $(\mathbf{8})$. Acredita-se que a elevada reatividade destes compostos ramificados é devida à ocorrência de rotâmeros estáveis que conduzem a uma orientação favorável para a ciclização intramolecular (Fredholt et al., 1995).

Todos os compostos também foram avaliados quanto às suas estabilidades enzimáticas em plasma humano a $40 \%$, homogenato de fígado de rato a $20 \%$ e homogenato de fígado de porco a $20 \%$, em todos os casos com ensaios realizados a $37^{\circ} \mathrm{C}$ e pH 7,4. Os resultados obtidos mostraram que os ésteres de paracetamol se apresentaram estáveis quando analisados em plasma humano. No entanto, para os ésteres de fenol, quando estudados em plasma humano, observou-se uma ligeira influência catalítica das enzimas plasmáticas sobre os compostos 13, 15 e 16. Por outro lado, para o composto $\mathbf{1 4}$, não se observou catálise enzimática.

Segundo os autores, com exceção do composto 10, todos os demais compostos sofreram acentuada degradação enzimática quando analisados em homogenato de fígado de rato e de porco. Assim, observou-se que a degradação destes compostos ocorreu através de uma ciclização intramolecular em tampão aquoso e em plasma humano, sendo que, neste último caso, se observa catálise enzimática pouco significativa. Já para os ensaios em homogenatos de fígado, observa-se que tais compostos são extensamente degradados pela via enzimática. Para o composto 10 , que se apresentou como uma exceção, o tempo de meia-vida 
obtido em tampão aquoso, plasma humano e homogenatos de fígado foi o mesmo. O diferente comportamento deste composto foi atribuído à presença da carga negativa na sua estrutura ao $\mathrm{pH}$ fisiológico - pois ésteres contendo cargas negativas próximas da ligação éster são freqüentemente pobres substratos para esterases (Nielsen, Bundgaard, 1987) - juntamente com o maior impedimento estérico na cadeia lateral do sistema transportador.

\section{O grupo amida como agente nucleofílico}

Com o objetivo de proteger fármacos fenólicos do efeito de primeira passagem, Fredholt e Bundgaard (1993) desenvolveram um sistema de potenciais pró-fármacos ativados pelo ataque nucleofílico do grupo amida - que em valores de $\mathrm{pH}$ mais elevados se encontra na forma de íon amidato - sobre a carbonila do grupo carbamato, que une o sistema transportador ao fármaco (Esquema 5).

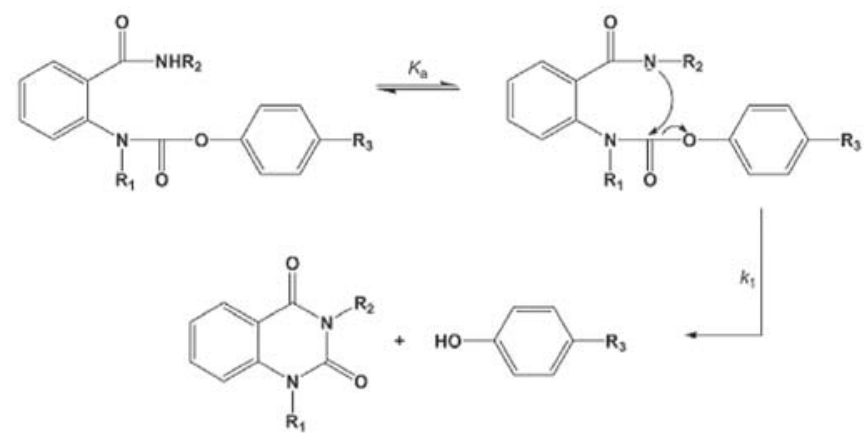

ESQUEMA 5 - Modelo de potencial pró-fármaco ativado por ciclização intramolecular a uma quinazolidinona, com concomitante liberação do fármaco fenólico, proposto por Fredholt e Bundgaard (1993).

De acordo com este modelo, uma larga variedade de compostos foi sintetizada e avaliada quanto à sua estabilidade in vitro. Os resultados dos estudos para a degradação de todos os compostos em tampão aquoso e em plasma humano a $40 \%$, ambos em pH 7,4 e $37^{\circ} \mathrm{C}$, são apresentados na Tabela II.

Pelos dados apresentados nesta Tabela, pode-se observar que a degradação dos compostos 17-33 foi influenciada fortemente pela natureza do grupo $\mathrm{R}_{2}$. Dentre os compostos mais estáveis à hidrólise química, pode-se contar os derivados 24 a 26. A elevada estabilidade destes três compostos - comparativamente aos demais derivados que possuíam um grupo metila ou etila na mesma posição - pode ser devida ao efeito estérico causado pelas suas cadeias mais volumosas. Por outro lado, a elevada reatividade de compostos tais como 27 e 28 pode ser atribuída ao efeito polar dos grupos éster e amida desses compostos, respec-
TABELA II - Compostos sintetizados por Fredholt e Bundgaard (1993) e respectivos tempos de meia-vida em tampão aquoso e plasma humano a $40 \%$, ambos a $37^{\circ} \mathrm{C}$ e $\mathrm{pH} \mathrm{7,4}$

\begin{tabular}{llcccc} 
& & & \\
\hline
\end{tabular}

${ }^{a}$ Os dados entre parênteses ou simplesmente marcados com o expoente "a" indicam os valores obtidos em plasma humano a $80 \%$; nd: valores não determinados.

tivamente, que poderão favorecer a desprotonação do nitrogênio amídico, convertendo este num nucleófilo mais eficaz (amidato), facilitando a ciclização intramolecular. Quanto à estabilidade enzimática, observa-se que todos os compostos foram sensíveis à hidrólise pelas enzimas plasmáticas, o que sugere que estes derivados são substratos das hidrolases do plasma humano.

A reatividade do derivado 20 foi avaliada em tampão aquoso numa escala de $\mathrm{pH}$ variando entre 6,5 e 10. Os resultados obtidos revelaram que este composto liberou o fenol quantitativamente através de uma ciclização intramolecular catalizada pelo íon hidróxido, por meio de um pré-equilíbrio de ionização do grupo $o$-amido, como descrito no Esquema 5. Por outro lado, a análise dos produtos de hidrólise do mesmo derivado, numa escala de $\mathrm{pH}$ variando entre 1 e 6 , mostrou que a liberação do fenol ocorreu pela ciclização a uma quinazolidinona e pela formação 
da respectiva benzamida (sistema transportador não ciclizado), como apresentado no Esquema 6.

Os dados experimentais mostraram, ainda, que a diminuição do $\mathrm{pH}$ conduziu a uma diminuição da concentração da quinazolidinona formada, apontando para a liberação do fenol por um mecanismo alternativo à ciclização. Esse mecanismo não pode ser atribuído à simples clivagem do carbamato por catálise ácida, uma vez que a degradação do derivado 20 é independente do $\mathrm{pH}$, no intervalo de pH entre 1 e 4,5. A variação da formação dos produtos pode, antes, ser atribuída à formação de um intermediário tetraédrico por um equilíbrio ácido-base, num passo limitante da velocidade da reação, para formar o fenol, a quinazolidinona e a respectiva benzamida, como apresentado no Esquema 6.

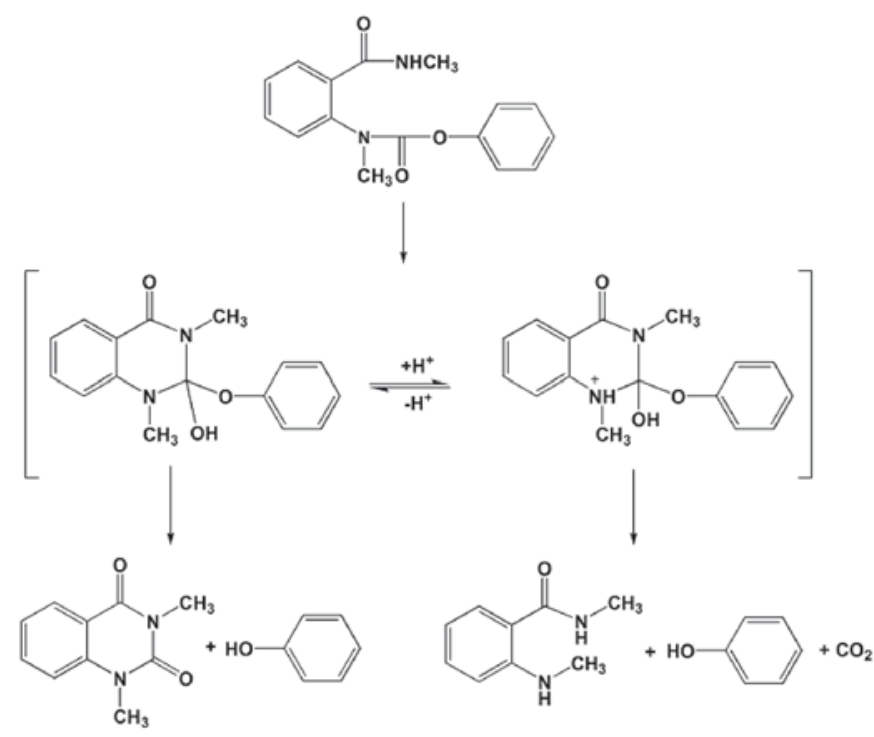

ESQUEMA 6 - Caminhos de liberação do fenol a partir do derivado 20 numa escala de $\mathrm{pH}$ variando entre 1 e 6 , segundo Fredholt e Bundgaard (1993).

Com o objetivo de aumentar a solubilidade em água de um inibidor da protease do VIH, o KNI-727 (34), diferentes trabalhos foram desenvolvidos utilizando-se o grupo amida como agente nucleofílico no desenvolvimento de prófármacos (Matsumoto et al., 2001, a,b), Tamamura et al. (1998)). Esses trabalhos apresentam um modelo de potenciais pró-fármacos ativados, em condições fisiológicas, pelo ataque nucleofílico do grupo amida à ligação éster que une o sistema transportador ao fármaco, como apresentado pelo Esquema 7.

Seguindo este modelo, um conjunto de derivados de 34 foi sintetizado (Tabela III) e analisado quanto à reatividade em solução aquosa de tampão fosfato isotônico, a $37^{\circ} \mathrm{C}$ e pH 7,4 .

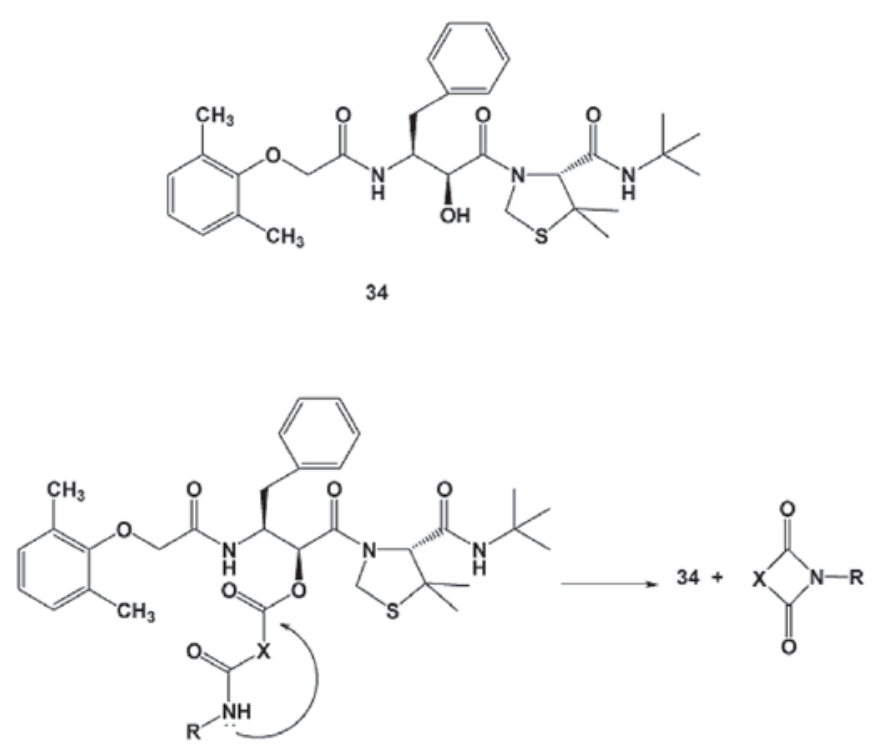

ESQUEMA 7 - Liberação do princípio ativo KNI-727 (34) a partir do modelo de Matsumoto et al. (2001a), através de uma ciclização intramolecular conseqüente à formação de uma imida, em que $\mathrm{X}=-\left(\mathrm{CH}_{2}\right)$ - e R é a parte hidrofílica do sistema transportador e varia entre ácidos carboxílicos alifáticos, amidas não-substituídas, cloretos de amônio derivados de aminas primárias e terciárias ou, ainda, o grupo fenila.

TABELA III - Derivados de KNI-727 sintetizados por Matsumoto et al. (2001a) e respectivos tempos de meiavida em tampão fosfato isotônico a $37^{\circ} \mathrm{C}$ e pH 7,4

\begin{tabular}{lccc}
\hline Composto & $\mathrm{X}$ & $\mathrm{R}$ & $\mathrm{t}_{1 / 2} / \mathrm{min}$ \\
\hline $\mathbf{3 5}$ & $-\left(\mathrm{CH}_{2}\right)_{2}{ }^{-}$ & $-\left(\mathrm{CH}_{2}\right)_{2} \mathrm{NH}_{2}$ & 21,0 \\
$\mathbf{3 6}$ & $-\left(\mathrm{CH}_{2}\right)_{3}{ }^{-}$ & $-\left(\mathrm{CH}_{2}\right)_{2} \mathrm{NH}_{2}$ & 1680,0 \\
$\mathbf{3 7}$ & $-\mathrm{CH}_{2} \mathrm{C}^{\left.-\mathrm{CH}_{3}\right)_{2}}$ & $-\left(\mathrm{CH}_{2}\right)_{2} \mathrm{NH}_{2}$ & 5,0 \\
$\mathbf{3 8}$ & $-\left(\mathrm{CH}_{2}\right)_{2}{ }^{-}$ & $-\mathrm{CH}_{2} \mathrm{COOH}$ & 1122 \\
$\mathbf{3 9}$ & $-\left(\mathrm{CH}_{2}\right)_{2}{ }^{-}$ & $-\left(\mathrm{CH}_{2}\right)_{2} \mathrm{COOH}$ & 1008 \\
\hline
\end{tabular}

Pela análise dos produtos de hidrólise, foi possível confirmar que a saída do grupo abandonador ocorreu de forma quantitativa, através de uma ciclização intramolecular conseqüente à formação da respectiva imida, como único caminho de liberação do princípio ativo. Nessas condições de análise, a reatividade de todos os compostos foi afetada, quer pela estrutura do espaçador (X no Esquema 7), quer pela estrutura da porção hidrofílica (R no Esquema 7). Assim, os tempos de meia-vida obtidos para degradação dos derivados de 34, nessas condições, variaram entre 5 e 1680 minutos. O tempo de meia-vida obtido para degradação do composto 35 , por exemplo, foi de 21 minutos (Tabela III). Por outro lado, o tempo de meia-vida 


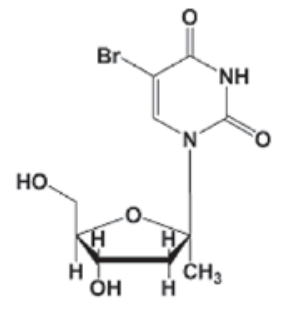

40

Observa-se, pela análise desta tabela, que a reatividade dos derivados 41-43 aumentou com o aumento do $\mathrm{pH}$. Essa evidência experimental pode ser explicada pelo fato do grupo amino se encontrar protonado em meio ácido, tornando-se um fraco nucleófilo. Um outro fator que influenciou a reatividade destes compostos foi a cadeia do sistema transportador. Observa-se que, em diferentes valores de $\mathrm{pH}$, o composto $\mathbf{4 2}$ se apresenta mais estável que o composto 41, possivelmente devido ao efeito estérico do grupo ciclo-hexila de $\mathbf{4 2}$ que causa diminuição da velocidade de ciclização do seu sistema transportador.

A análise dos produtos de degradação de $43 \mathrm{em} \mathrm{pH}$ 7,4 e $37^{\circ} \mathrm{C}$ revelou que a liberação de $\mathbf{4 0}$ ocorre através de uma ciclização intramolecular conseqüente à respectiva piperazinona 44 , com um rendimento de $95,2 \%$. Por outro lado, os resultados obtidos pela degradação de 42 e 43 em plasma de rato mostraram que estes compostos são bons substratos para as esterases aí presentes. No entanto, os mesmos compostos não se apresentam como substratos para as enzimas do plasma humano.

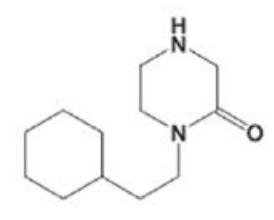

44

\section{CONCLUSÕES}

O desenvolvimento de pró-fármacos mostra-se como uma estratégia muito útil, quando se pretende melhorar as qualidades farmacocinéticas e farmacodinâmicas do fármaco. Em particular, a utilização de pró-fármacos ativados pela via intramolecular é uma estratégia que conduz à liberação do princípio ativo independentemente da atividade enzimática nos organismos animais, pois a liberação do fármaco ocorre quimicamente através do ataque de um grupo nucleofílico sobre um grupo eletrofílico, de modo a produzir uma estrutura cíclica (geralmente derivada do sistema transportador) e uma unidade parental (geralmente, o próprio princípio ativo). Este tipo de estratégia mostra-se como um meio útil de controlar a liberação do princípio ativo pela modulação química da estrutura do sistema transportador. Assim, a ciclização intramolecular apresenta-se como um excelente meio para a liberação, de forma controlada, de uma larga variedade de fármacos.

Os fármacos são compostos que contam, de um modo geral, com diferentes grupos funcionais na sua estrutura molecular. No desenvolvimento de pró-fármacos ativados pela via intramolecular, a escolha do grupo funcional no qual ocorrerá a derivatização é uma tarefa de grande importância. A velocidade de liberação do fármaco a partir de um sistema transportador apresenta-se, para além de outros fatores, como uma função do $\mathrm{p} K$ a do grupo abandonador (geralmente, o princípio ativo). Além disso, muitas vezes a derivatização em determinado grupo funcional pode evitar transformações metabólicas indesejáveis envolvendo este grupo. Muito importante, também, é a escolha da unidade transportadora. Esta unidade, quando ligada ao fármaco deve obedecer a alguns requisitos, tais como: elevada estabilidade frente a enzimas dos organismos animais, de modo a não comprometer o processo e, com isso, a velocidade de liberação do princípio ativo, e elevada solubilidade no suco gástrico. O desenvolvimento de modelos de pró-fármacos com essas características poderá conduzir à obtenção de um tipo de pró-fármaco ideal, genericamente aplicável a diferentes fármacos.

\section{ABSTRACT}

\section{Intramolecular cyclization: a promising strategy in the prodrug conception}

Many drugs used for the treatment of common diseases are associated with various adverse effects and limited bioavailability. The suppression of such problems continues to be a very important target for scientists. The development of prodrugs for controlled release in vivo is an attractive way to overcome these problems. Classical in prodrug is an inactive molecule which the parent drug is covalently bonded to a carrier unit, and which can liberate the drug through chemical or enzymatic pathways. A new and interesting prodrug system for amine, alcohol, and thiol drugs takes advantage of several easy intramolecular cyclization reactions. So, the cyclization process can control the release rate of the parent drug. In this paper is a review about the prodrug strategies based on intramolecular cyclization reactions is presented.

UNITERMS: Prodrugs. Intramolecular cyclization. Drugs/controlled release. 


\section{AGRADECIMENTOS}

Aos Professores Paula Gomes, do Centro de Investigação em Química da Universidade, do Porto e Rui Moreira do Centro de Ciências Farmacêuticas da Universidade de Lisboa (Portugal), pelas ricas discussões desenvolvidas no contexto dos Pró-fármacos ativados por ciclização intramolecular, e às Professoras Madalena Areias, do Departamento de Química Fundamental da Universidade Federal de Pernambuco e Elisângela Mendonça da Universidade Estadual da Paraíba pela forte contribuição na revisão do texto após tradução para o português brasileiro.

\section{REFERÊNCIAS BIBLIOGRÁFICAS}

ALBERT, A. Chemical aspects of selective toxicity. Nature, v.182, p.421-422, 1958.

ANDERSEN, R.; NIELSEN, C.; BEGTRUP, M.; STEEN, F.; BRODIN, J.; FROKJAER, S.; STEFFANSEN, B. In vitro evaluation of $\mathrm{N}$-methyl amide tripeptidomimetics as substrates for the human intestinal di-/tri-peptide transporter hPEPT1. Eur. J. Pharm. Sci., v.28, p.325-335, 2006.

BUNDGAARD, H. Novel Chemical approaches in prodrug design. Drugs Future., v.16, p.443-458, 1991.

BUNDGAARD, H.; FALCH, E.; LARSEN, C.; MIKKELSON, T. Pilocarpine prodrugs I. Synthesis, physicochemical properties and kinetics of lactonization of pilocarpic acid esters. J. Pharm. Sci., v.75, p.36-43, 1986.

BRUICE, T. C.; PANDIT, U. K. Intramolecular models depicting the kinetic importance of "fit" in enzymatic catalysis. PNAS, v.46, n.4, p.402-404, 1960.

CARVALHO, E.; FRANCISCO, A.; ILEY, J.; ROSA, E. Triazene drug metabolites. Part 17: synthesis and plasma hydrolysis of acyloxymethyl carbamate derivatives of antitumour triazenes. Bioorg. Med. Chem., v.8, p.1719$1725,2000$.

CHUNG, M.; FERREIRA, E. O processo da latenciação no planejamento de fármacos. Quim. Nova, v.22, p.75-84, 1999.

DECHTLARUK, W.; JOHNSON, G.; SOLOMON, H. Gaschromatographic method for acetaminophen ( $N$-acetyl- $p$ aminophenol) based on sequential alkylation. Clin. Chem., v.22, p.879-883, 1976.
DE VRUEH, R. L. A.; SMITH, P. L.; LEE, C. P. Transport of L-Valine-Acyclovir Via the Oligopeptide Transporter In The Human Intestinal Cell Line, Caco-2. J. Pharmacol. Exp. Ther., v.286, p.1166-1170, 1998.

ETTMAYER, P.; AMIDON, G. L.; CLEMENT, B.; TESTA, B. Lessons learned from marketed and investigational prodrugs. J. Med. Chem., v.47, p.2393-2404, 2004.

FREDHOLT, K.; BUNDGAARD, H. Cyclization-activated phenyl carbamate prodrug forms for protecting phenols against first-pass metabolism. Int. J. Pharm., v.91, p.3949, 1993.

FREDHOLT, K.; MORK, N.; BEGTRUP, M. Hemiesters of aliphatic dicarboxylic acids as cyclization-activated prodrug forms for protecting phenols against first-pass metabolism. Int. J. Pharm., v.123, p.209-216, 1995.

FRIEDRICHSEN, G.; JAKOBSEN, P.; TAUB M.; BEGTRUP, M. Application of enzymatically stable dipeptides for enhancement of intestinal permeability: synthesis and in vitro evaluation of dipeptide-coupled compounds. Bioorg. Med. Chem., v.9, p.2625-2632, 2001.

GUETTARI, N.; LOUBIÈRE, L.; BRISSON, E.; KLATZMANN, D. Use of Herpes Simplex Virus Thymidine Kinase to Improve the Antiviral Activity of Zidovudine Virology, v.235, p.398-405, 1997.

GUO, X.; TUNG, M. L.; CHEN, H. X.; CHANG, C. N.; ZHU, J. L.; CHANG, C. P.; PIZZORNO, G.; LIN, T. S.; CHENGT, Y. 5-fluoro-2-pyrimidinone, a liver aldehyde oxidase-activated prodrug of 5-fluorouracil. C. Biochem. Pharmacol., v.49, p.1111-1116, 1995.

HAN, H. K.; VRUEH, R. L. A.; RHIE, J. K.; COVITZ, K. M. Y.; SMITH, P. L.; LEE, C. P.; OH, D. M.; SADÉE, W.; AMIDON, G. 5'-Amino acid esters of antiviral nucleosides, acyclovir, and AZT are absorbed by the intestinal PEPT1 peptide transporter. L. Pharm. Res. v.15, p.1154-1159, 1998.

HEYDEN, S.; HGHLEY, M.; BRUIJN, E.; TJADEN, U.; REEUWIJK, H.; SLOOTEN, H.; OOSTEROM, A.; MAES, R. Pharmacokinetics and bioavailability of oral 5'-deoxy-5-fluorouridine in cancer patients. Br. J. Clin. Pharmacol., v.47, p.351-356, 1999. 
KOVACH, I.; PITMAN, I.; HIGUCHI, T. Amino acid esters of phenols as prodrugs: Synthesis and stability of glycine, $\boldsymbol{b} \boldsymbol{\partial}$-aspartic acid, and $\boldsymbol{a} \boldsymbol{\delta}$-aspartic acid esters of pacetamidophenol. J. Pharm. Sci., v.70, p.881-885,1981.

LARSEN, S.; ANKERSEN, M.; LARSEN, C. Kinetics of degradation and oil solubility of ester prodrugs of a model dipeptide (Gly-Phe). Eur. J. Pharm. Sci., v.22, p.399-408, 2004.

LYNX, M.; MCKEE, E. 32 -Azido-32 -deoxythymidine (AZT) is a competitive inhibitor of thymidine phosphorylation in isolated rat heart and liver mitochondria. Biochem. Pharmacol., v.72, p.239-243, 2006.

MATSUMOTO, H.; SOHMA, Y.; KIMURA, T.; HAYASHI, Y.; KISO, Y. Controlled drug release: new water-soluble prodrugs of an HIV protease inhibitor. Bioog. Med. Chem. Lett., v.11, p.605-609, 2001a.

MATSUMOTO, H.; KIMURA, T.; HAMAWAKI, T.; KUMAGAI, A.; GOTO, T.; SANO, K.; HAYASHI, Y.; KISO, Y. Design, synthesis, and biological evaluation of anti-HIV double-drugs: conjugates of HIV protease inhibitors with a reverse transcriptase inhibitor through spontaneously cleavable linkers. Bioorg. Med. Chem., v.9, p.1589-1600, 2001b.

MICHIGAN STATE UNIVERSITY: IONIZATION CONSTANTS OF HETEROATOM ORGANIC ACIDS. Disponível em: <http://www.cem.msu.edu/ $\sim$ reusch/VirtualText/acidity2.htm>. Acesso em: 01 out. 2007

NIELSEN, N.; BUNDGAARD, H. Prodrugs as drug delivery systems. 68. Chemical and plasma-catalyzed hydrolysis of various esters of benzoic acid: a reference system for designing prodrug esters of carboxylic acid agents. Int. J. Pharm., v.39, p.75-85, 1987.

OTTO, M. New nucleoside reverse transcriptase inhibitors for the treatment of HIV infections. Curr. Opin. Pharmacol., v.4, p.431-436, 2004.

PETERSON LAB - PENN STATE DEPARTMENT OF CHEMISTRY. Diponível em: <http:// research.chem.psu.edu/brpgroup/pKa_compilation.pdf $\$$. Acesso em: 01 out. 2007.
SAARI, W.; SCHWERING, J.; LYLE, P.; SMITH, S.; ENGENLHARDT, E. Cyclization-activated produgs. Basic esters of 5-bromo-2'-deoxyuridine. J. Med. Chem., v.33, p.2590-2595, 1990.

SHAN, D.; NICOLAOU, M. G.; BORCHARDT, R. T.; WANG, B. Prodrug strategies based on intramolecular cyclization reactions. J. Pharm. Sci., v.86, p.765-767, 1997.

SICCARDI, D.; GUMBLETON, M.; OMIDI, Y.; MCGUIGAN, C. Stereospecific chemical and enzymatic stability of phosphoramidate triester prodrugs of d4T in vitro. Eur. J. Pharm. Sci., v.22, p.25-31, 2004.

SONG, X.; LORENZI, P. L.; LANDOWSKI, C. P.; VIG, B. S.; HILFINGER, J. M. AND AMIDON, G. Amino Acid Ester Prodrugs of the Anticancer Agent Gemcitabine: Synthesis, Bioconversion, Metabolic Bioevasion, and hPEPT1-Mediated Transport. Mol. Pharm., v.2, p.157167, 2005.

SRIRAM, D.; YOGEESWARI, P.; SRICHAKRAVARTHY, N.; BAL, T. Synthesis of stavudine amino acid ester prodrugs with broad-spectrum chemotherapeutic properties for the effective treatment of HIV/AIDS. Bioorg. Med. Chem. Lett., v.14, p.1085-1087, 2004.

STEINGRIMSDOTTIR, H.; GRUBER, A.; PALM, C.; GRIMFORS, G.; KALIN, M.; EKSBORG, S. Bioavailability of aciclovir after oral administration of aciclovir and its prodrug valaciclovir to patients with leukopenia after chemotherapy. Antimicrob. Agents Chemother., v.44, p.207-209, 2000.

STEFFANSEN, B.; NIELSEN, C.; FROKJAER, S. Delivery aspects of small peptides and substrates for peptide transporters. Eur. J. Pharm. Biopharm., v.60, p.241-245, 2005.

TAMAMURA, H.; ISHIHARA, T.; OYAKE, H.; IMAI, M.; OTAKA, A.; IBUKA, T.; ARAKAKI, R.; NAKASHIMA, H.; MURAKAMI, T.; WAKI, M.; MATSUMOTO, A.; YAMAMOTOC, N.; FUJII, N. Convenient one-pot synthesis of cystine-containing peptides using the trimethylsilyl chloride-dimethyl sulfoxide/trifluoroacetic acid system and its application to the synthesis of bifunctional anti-HIV compounds. $J$. Chem. Soc., Perkin Trans., v.1, p.495-500, 1998. 
TESTA, B.; MAYER, J. Design of intramolecularly activated prodrugs. Drug Metab. Rev., v.30, p.787-807, 1998.

THOMSEN, K.; BUNDGAARD, H. Cyclization-activated phenyl carbamate prodrug forms for protecting phenols against first-pass metabolism. Int. J. Pharm., v.91, p.3949, 1993.

THOMSEN, A.; CHRISTENSEN, M.; BAGGER, M.; STEFFANSEN, S. Acyclovir prodrug for the intestinal di/ tri-peptide transporter PEPT1: comparison of in vivo bioavailability in rats and transport in Caco-2 cells. Eur. J. Pharm. Sci., v.23, p.319-325, 2004.

THOMSEN, A. E.; FRIEDRICHSEN, G. M.; SORENSEN, A. H.; ANDERSEN, R.; NIELSEN, C. U.; BRODIN, B.; BEGTRUP, M.; FROKJAER, S.; STEFFANSEN, B. Prodrugs of purine and pyrimidine analogues for the intestinal di/tri-peptide transporter PepT1: affinity for hPepT1 in Caco- 2 cells, drug release in aqueous media and in vitro metabolism. J. Control. Release, v.86, p.279292, 2003.
VENNERSTROM, J.; NUZUM, E.; MILLER, R.; DORN, A.; GERENA, L.;. DANDE, P.; WILLIAM, E. RIDLEY, R.; MILHOUS, W. 8-Aminoquinolines Active against Blood Stage Plasmodium falciparum In Vitro Inhibit Hematin Polymerization. Antimicrob. Agents Chemother. v.43, p.598-602, 1999.

VIGROUX, A.; BERGON, M.; ZEEDE, C. Based on RingOpened Derivatives of Active Benzoxazolones and Oxazolidones as Mutual Prodrugs of Acetamiophen. $J$. Med. Chem., v.38, p.3983-3994, 1995.

ZAMBONIN, C. G.; ARESTA, A.; GRANO, M. In vitro toxicity of N3-methyl-52 -deoxy-5-fluorouridine, a novel metabolite of doxifluridine: a bioanalytical investigation. J. Pharm. Biomed. Anal., v.17, p.11-16, 1998.

ZHENG, A.; WANG, W.; ZHANG, H.; WANG, B. Two new improved approaches to the synthesis of coumarin-based prodrugs. Tetrahedron, v.55, p.4237-4254, 1999.

ZINUTTI, C.; HEYOB, M. B.; HOFFMAN, M.; MAINCENT, P. In-vivo evaluation of sustained release microspheres of 5-FU in rabbits. Int. J. Pharm., v.166, p.231-234, 1998.

Recebido para publicação em 21 de novembro de 2007 Aceito para publicação em 13 de março de 2008 